\title{
Interrelación actual entre Gobierno Corporativo, Respondabilidad y Auditoría Integral: una visión reformulada
}

\author{
Current relationship between Corporate Governance, Accountability \\ and Integral Audit: a reformulated vision.
}

\section{Carlos Mauricio De La Torre Lascano*}

\section{RESUMEN}

La búsqueda de rentabilidad por parte de las organizaciones frente a un mercado cada vez más globalizado, en donde el apetito de riesgo y el nivel de competitividad ascienden en forma desenfrenada, han ocasionado que las actividades desempeñadas por auditoría (interna o externa) se vean relegadas o sean insuficientes ante la escalada de hechos que tienen como constante la corrupción y la falta de ética empresarial. Por lo tanto, el objetivo del presente artículo se basó en la descripción de la interrelación que tiene la Auditoría Integral frente a la adopción de un sistema idóneo de gobierno corporativo, teniendo implícito la rendición de cuentas a todos los involucrados tanto internos como externos en el sistema, aplicando un enfoque cualitativo de los diferentes estándares internacionales emitidos por los organismos como AICPA (2016), Cadbury Committee (1992), COSO (2013), OCDE (2016), así como de diversos estudios doctrinales realizados sobre esta materia por autores como Arellano (2017), Englund, Gerdin y Abrahamsson (2013), Caridad, Hernández y De Pelekais (2014), GarcíaMarzá (2017), Dai y Vasarhelyi (2016), Claessens y Yurtoglu (2012), entre otros. Los estándares internacionales buscan fortalecer la transparencia en las organizaciones, necesaria para el desempeño de sus operaciones que beneficia a la sociedad en donde estas desarrollan sus actividades mercantiles. No obstante, el desafío que tiene Auditoría Integral es mayúsculo en torno los hechos de corrupción acaecidos. Siendo así, resulta necesario realizar una descripción que contribuya a verificar el rumbo adecuado para que Auditoría Integral aporte a generar valor y robustecer la transparencia en las organizaciones. El estudio realizado demuestra que existe una interrelación inherente entre los sistemas con base a los cuales las organizaciones son

\footnotetext{
* Doctor (Ph.D.) por la Universidad de Salamanca, Programa doctoral Estado de Derecho y Gobernanza Global. Magíster en Gerencia Empresarial, MBA. Mención Gerencia Financiera. Auditor por la Universidad Central del Ecuador. Diploma Superior en Docencia Universitaria. Profesor Titular e Investigador acreditado de la Universidad Central del Ecuador, Facultad de Ciencias Administrativas. Ecuador. Correo electrónico: cdelatorre@ uce.edu.ec
}

492 
administradas y la rendición de cuentas, obligatoria para fomentar la transparencia, que debe ser evaluada y monitoreada mediante la aplicación permanente y visión reformulada de Auditoría Integral.

Palabras clave: auditoría integral, corrupción, gobierno corporativo, respondabilidad, transparencia.

RECIBIDO: Junio 2018

ACEPTADO: Agosto 2018

\section{ABSTRACT}

The search of profitability by the organizations in front of an increasingly globalized market, where the risk appetite and the level of competitiveness ascend in an unrestrained way, have caused that the activities carried out by audit (internal or external) are impaired or are insufficient in the face of the escalation of events that have corruption as a constant and lack of business ethics. Therefore, the aim of the current paper was based on the description of relationship that Integral Audit has towards the adoption of an appropriate system of corporate governance, implying accountability to all those involved, both internal and external in the system, applying a qualitative approach to the different international standards issued by organizations such as AICPA (2016), Cadbury Committee (1992), COSO (2013), OECD (2016), as well as various doctrinal studies conducted on this subject by authors such as Arellano (2017), Englund, Gerdin y Abrahamsson (2013), Caridad, Hernández y De Pelekais (2014), GarcíaMarzá (2017), Dai y Vasarhelyi (2016), Claessens y Yurtoglu (2012), among others. The international standards seek to strengthen transparency in organizations, necessary for the performance of their operations that benefits the society where they develop their commercial activities. However, the challenge of Integral Audit is huge around the acts of corruption that have taken place. Thus, it is necessary to make a description that contributes to verify the appropriate course so that Integral Audit contributes to generate value and strengthen transparency in organizations. The study shows that there is an inherent interrelation between the systems on the basis of which organizations are managed and accountability, mandatory to promote transparency, but that it must be evaluated and monitored through the permanent application and reformulated vision of Integral Audit.

Key words: integral audit, corruption, corporate governance, accountability, transparency.

\section{Introducción}

Los accionistas de las compañías buscan que sus inversiones retornen en un tiempo prudencial, en mayor medida que la financiación inicial y en esa búsqueda de rentabilidad, el apetito de riesgo que asumen los administradores es un tema por el cual 
Interrelación actual entre Gobierno Corporativo, Respondabilidad y Auditoría Integral: una visión reformulada

deben responder en función de la gestión realizada. Asegurar al inversor que el capital disponible para las empresas en forma de acciones se administrará de tal manera que garantice el mayor rendimiento es esencial para el desarrollo del mercado de valores como alternativa al financiamiento de la empresa (Bortolon et al., 2013), es decir que la organización cuente con liquidez o a su vez sus acciones gocen de credibilidad y puedan ser ofertadas a buen precio, se enmarca en ello. Para lo cual deben adoptar marcos normativos que les permitan alcanzar los objetivos planteados para ser administradas de una manera adecuada, sus actividades tanto administrativas, como de orden operativo deberían estar enmarcadas dentro de valores éticos.

De manera retrospectiva, se puede afirmar que a medida que la revolución industrial tomó impulso en el siglo XIX, las transacciones se volvieron más sofisticadas, mucho más frecuentes, alcanzando niveles internacionales, incrementándose considerablemente el número de operaciones. Las empresas que fueron administradas de manera competente, en consecuencia, se volvieron más prósperas y eso significaba que los propietarios de las empresas necesitaban contratar contadores para asegurarles que eran rentables y solventes (Donleavy, 2016). En la actualidad, la globalización y los avances tecnológicos son hechos que repercuten en las economías del mundo con gran nivel de incidencia en todas las organizaciones y arreglos institucionales que se expresan en la emisión de estándares internacionales para mermar los crecientes escándalos en las esferas pública y privada, ha sido una constante a nivel global.

Maldonado (2016), argumenta que es sorprendente el nivel de irregularidades o esquemas de corrupción en las que no son solo protagonistas multinacionales o altos funcionarios públicos, sino que ésta involucra a entidades que cualquier ciudadano común no creería como el caso de los fondos del Banco del Vaticano, el escándalo en el seno de la FIFA, el financiamiento a grupos irregulares, narcoterroristas, en extremo peligrosos y recientemente los Panama Papers y el esquema de corrupción fraguado por la constructora brasileña Odebretch en el que siguen descubriéndose actores de alto rango político que han sido cesados de sus cargos, encarcelados o se encuentran 
prófugos. El rol de auditoría frente a estos hechos sigue siendo cuestionado. En tanto que la demanda de ética en los negocios está creciendo en el mundo. Se espera ética de los empresarios y las empresas, pero también de los políticos y los responsables gubernamentales, al ser quienes determinan la normativa que rige el accionar de las instituciones. Esta demanda forma parte de un sentimiento profundo y generalizado a nivel mundial. Existe la percepción de que el debate ético en general ha sido marginado de la agenda colectiva (Kliksberg, 2012).

La problemática por la falta de adopción de estándares internacionales en las organizaciones, que generen una confianza real en dichos marcos por su aporte de manera efectiva al desarrollo de las operaciones, desemboca en el cometimiento de acciones fraudulentas de todo orden a escala mundial, situación que se agrava debido a la falta de transparencia por parte de sus directivos y alta gerencia. "La gerencia necesita ser sensible a las informaciones que dinamizan la organización en todos sus niveles" (Yoris, 2017, p. 337). Se requiere entonces, que los sistemas por los cuales las organizaciones son administradas y controladas tengan un nuevo enfoque en el contexto actual que debe abordar auditoría integral. Es decir, el escenario donde las organizaciones desarrollan sus actividades debe ser redefinido, bajo estándares de general aceptación, la necesidad de transparencia mediante la rendición de cuentas frente a las actividades de aseguramiento ejercidas por auditoría, con una visión actual de esa interrelación existente generan la necesidad de estudio sobre este tema.

\section{Aspectos metodológicos}

El presente trabajo se desarrolló con base a un estudio cualitativo exploratorio de las diferentes definiciones emitidas por organismos internacionales especializados en Auditoría, que buscan incrementar la transparencia de las organizaciones mediante su correcta aplicación y rendición de cuentas oportuna, todo ello bajo la adecuada supervisión de auditoría. Se revisaron varias fuentes documentales, especialmente estudios realizados por académicos; que incluyen las investigaciones elaboradas para 
Interrelación actual entre Gobierno Corporativo, Respondabilidad y Auditoría Integral: una visión reformulada

determinar dicho impacto. Aunque existe vasto cúmulo de información sobre gobierno corporativo y respondabilidad, la descripción se ha enfocado sobre aquella que tiene correspondencia con la relevancia de una adecuada función de auditoría, ya que ello constituye la base del objetivo central del estudio, que busca describir la interrelación de Auditoría Integral en torno a la adopción de un sistema adecuado de gobierno corporativo que abarque rendición de cuentas a los protagonistas internos y externos del sistema. Es necesario acotar que, al tratarse de un estudio cualitativo documental, la técnica de la descripción, basado en la recopilación de doctrina y normativa internacional en torno a la materia, es el más idóneo.

\section{Fundamentación teórica}

Debido a que el objetivo general que buscó el estudio realizado radicó en determinar la interrelación que tiene Auditoría Integral frente a la adopción de un sistema idóneo de gobierno corporativo, teniendo implícito la rendición de cuentas en base a preceptos internacionales, se procedió a la siguiente argumentación detallada en esta sección:

\section{1.) Gobierno Corporativo}

El desarrollo del gobierno corporativo ha tenido gran trascendencia en el transcurso de los últimos veinticinco años, por lo que se puede aseverar que no es reciente, pero sí desempeña un rol preponderante dentro de los estándares internacionales vigentes. La falta de confianza de los inversionistas en la honestidad y responsabilidad de las compañías cotizadas en el mercado de valores del Reino Unido, debido a escándalos de grupos empresariales por fallas del gobierno corporativo en la década de 1980, fue el estímulo para la creación de un comité que regule dichas desviaciones. El aspecto determinante del enfoque fue la introducción de un conjunto de recomendaciones o disposiciones enumeradas en un código sobre principios de buenas prácticas comúnmente reconocidas sobre diversos aspectos de gobierno 
corporativo, como la estructura del consejo, composición del comité e independencia (Arcot, Bruno y Faure-Grimaud, 2010).

Desde su aparición formal con la emisión del reporte del Comité Cadbury en 1992, donde se establecieron las bases para la emisión de tres principios fundamentales: i) principio de transparencia, ii) integridad, y iii) rendición de cuentas; que debían ser cumplidos por entidades listadas en la London Stock Exchange, que a su vez fueron estipulados y extendidos también a las organizaciones del sector público en 2001 por la International Federation of Accountants (IFAC por sus siglas en inglés), hasta la actualidad. Su definición involucra a varios protagonistas dentro y fuera de las organizaciones, determinando directrices desde diferentes aristas para la consecución de los objetivos organizacionales. El pasado reciente (y todos los escándalos recurrentes a lo largo de la historia empresarial) nos han dejado como aprendizaje que una adecuada toma de decisiones es producto de la virtud ética individual y organizacional. La mayoría de las personas no se involucran deliberadamente en malas prácticas, sino las estructuras que los rodean pueden conducirlos en esa dirección y colocar enormes costos en el individuo que rechaza (Gunz, 2012).

En torno a su definición, básicamente se entiende que "gobierno corporativo es el sistema por el cual las compañías son dirigidas y controladas" (Cadbury Comitte, 1992, p. 14). Desde el punto de vista financiero, en donde los gerentes administren adecuadamente el capital suministrado y no lo inviertan en proyectos que no sean rentables, Shleifer y Vishny (1997) determinan que el "gobierno corporativo se ocupa de las formas en que los proveedores de financiación a las empresas se aseguran de obtener un rendimiento de su inversión" (p. 737). Esta definición puede ampliarse para definir al gobierno corporativo como un asunto relacionado con la resolución de problemas de acción colectiva entre diversos inversionistas y la conciliación de conflictos de interés entre varios titulares de los derechos corporativos (Claessens y Yurtoglu, 2012). 
Interrelación actual entre Gobierno Corporativo, Respondabilidad y Auditoría Integral: una visión reformulada

Con una perspectiva más holística, Mostepaniuk (2017), determina que el gobierno corporativo es un proceso complejo que involucra herramientas organizacionales, legales, económicas, motivacionales y sociales, cuya combinación proporciona un ambiente de trabajo único que permite minimizar los costos al reducir la brecha entre los intereses de los administradores y propietarios. La Organización para la Cooperación y el Desarrollo Económicos (OCDE) considera que uno de los aspectos esenciales del gobierno corporativo consiste en garantizar la circulación de capital externo hacia las empresas, tanto en forma de capital como de deuda (OCDE, 2016), esto implica encontrar diversas maneras de motivar a las diferentes partes interesadas en la organización para que alcancen niveles óptimos tanto de capital económico y talento humano.

Por lo expuesto, se podría aseverar entonces que gobierno corporativo es el conjunto de políticas formales e informales que regulan las relaciones entre la organización y todos aquellos que tienen intereses (stakeholders) sobre la misma. Entre estos puede señalarse a: el Estado para la consecución de recursos mediante impuestos, accionistas para recibir el fruto de sus inversiones, proveedores mediante la entrega de materiales para que produzcan sus mercaderías, clientes por efectos de calidad, la sociedad mediante la contribución de fuentes de empleo y cuidado del ambiente, funcionarios por el desarrollo de su trayectoria profesional, bajo un marco muy bien delineado para la consecución de objetivos comunes (De La Torre, 2017). Por lo que resulta lógico entender que exista una relación inmanente entre ética y gobierno corporativo.

\section{2.) Respondabilidad - Rendición de cuentas - Accountability}

La literatura especializada en esta temática reconoce el término en inglés (accountability) por ser de procedencia anglosajona. La respondabilidad, rendición de cuentas, accountability se puede definir como "ser responsable", es decir, poder realizar rendición de cuentas. En el entorno corporativo o ámbito empresarial (objeto del 
presente estudio), se ha asociado este término estrechamente con la auditoría financiera y la elaboración de informes, así como con la contabilidad en general. Dentro de la concepción neoclásica de la corporación, se describe en términos de los deberes que se deben a sus accionistas, los diferentes aportes realizados sobre la materia han llevado a una mayor conciencia de los deberes asociados con las diversas otras relaciones en las que las empresas suelen participar. Desde esta perspectiva, las organizaciones están obligadas a reconocer su respondabilidad ante una red más amplia de partes interesadas o stakeholders (Kolb, 2008).

Desde el punto de vista económico, Díaz et al. (2013) determinan que la respondabilidad financiera es la obligación de quienes administran fondos o bienes sobre los cuales tienen obligaciones de rendir cuentas en el desempeño de sus funciones, en un marco de transparencia financiera, tanto a los directivos, empleados públicos, así como a miembros de la sociedad civil. Según Rozas (1998) el origen de la respondabilidad data de 1975 , habría sido creado y propuesto por la Contraloría General de Canadá. Su relación con Auditoría es intrínseca, toda vez que el proceso de auditoría contiene una concordancia con la responsabilidad, acotando que la responsabilidad primaria de Auditoría Financiera es la de emitir una opinión acerca de la razonabilidad de los estados financieros en su conjunto frente a normatividad internacional vigente, es decir el auditor debe establecer en su informe si la información revelada en los estados contables es correcta y obedece a preceptos adoptados internacionalmente con cumplimiento de normativa legal.

Torres y Negrón (2016) definen a la responsabilidad gerencial como el "proceso de rendición de cuentas que integra el ámbito económico de una organización pública o privada, con aspectos medioambientales y sociales, bajo un enfoque ético, de sostenibilidad y de calidad" (p. 138), entre otros los factores que han fomentado la aceptación global de estos preceptos son: expectativas de la ciudadanía en todos los estratos económicos, múltiples y variadas presiones sociales que provienen de diferentes fundaciones y organismos, preocupación por el deterioro del medioambiente 
Interrelación actual entre Gobierno Corporativo, Respondabilidad y Auditoría Integral: una visión reformulada

en donde desarrollan las actividades industriales con énfasis en los grandes grupos multinacionales, necesidad de combatir el azote generalizado y más frecuente de la corrupción a través de una gestión transparente. Con este panorama mucho más amplio en torno al alcance de la respondabilidad gerencial, Caridad et al. (2014) consideran que ésta, "ya no solamente sería un acto de rendir cuentas a la autoridad superior, sino también al ciudadano a ser contemplado en su operacionalización" (p. 37).

El fenómeno de la corrupción no solo se oculta en complicadas operaciones de ingeniería financiera aplicadas por grandes grupos corporativos multinacionales, sino que se materializa debido a normativas blandas y permisivas en los países, ante lo cual las corporaciones alegan justificaciones de diverso orden, sumándose la clara falta de voluntad política para reprimir este flagelo que devasta a las naciones. Una regulación robusta tendiente a fortalecer los esquemas de transparencia institucional contribuye a mejorar la integridad empresarial. Søreide (2006), señala el riesgo que transacciones comerciales internacionales corruptas se incremente se debe a dos razones: a) la probabilidad que las multinacionales y sus directivos sean objeto de enjuiciamiento local en los países donde se ejecutan es frecuentemente baja, ya que la corrupción es generalizada y los funcionarios públicos son sobornables, y b) los tratados internacionales más importantes que se ocupan de la corrupción transfronteriza tienen varias debilidades y no han sido suscritos por muchos países. "Los esfuerzos más recientes se han centrado en combatir la corrupción a través de medidas de transparencia y rendición de cuentas" (Fukuyama, 2017, p. 58). Resulta necesario destacar que en estos procesos el rol de la sociedad civil es clave, toda vez que ésta exige transparencia en el accionar de empresarios, funcionarios públicos o políticos.

\section{3.) Auditoría Integral}

Las compañías son creadas con la finalidad de conseguir ganancias, es decir el espíritu con el que son diseñadas obedece a la obtención de rentabilidad. Para la consecución de tal premisa, las organizaciones preparan toda una compleja estructura 
que obviamente incluye la adquisición de recursos y el establecimiento de objetivos que les permitirá alcanzar dichos réditos en función de su apetito de riesgo, por lo que deben adoptar marcos normativos ya sean de orden legal o de general aceptación. "El control interno permite que una organización gestione con mayor eficacia los cambios que se produzcan dentro del entorno económico y competitivo, la dirección de la organización, así como sus prioridades y modelos de negocios cambiantes" (COSO, 2013, p. 1). Siendo así, los controles correctivos, como la auditoría interna, externa, procesos de revisión realizados por la alta gerencia con un alto grado de independencia entre los actores involucrados en los procesos administrativos y contables de la empresa, deben ser orientados a determinar los riesgos asociados a la rendición de cuentas buscando la transparencia de dichos resultados, obteniendo accountability dentro del marco financiero de las organizaciones (García et al., 2014).

"El control es considerado en la filosofía empresarial moderna como el análisis permanente de las desviaciones entre objetivos y realizaciones, y la adopción de las medidas correctoras que permitan cumplir con los objetivos o bien su adaptación necesaria" (Obispo y Gonzales, 2015, p. 67). Entendiendo que actualmente el control es un proceso continuo que debe agregar valor a la institución, sin descartar la segregación clásica del control en: previo, concurrente y posterior. Para el correcto funcionamiento de los sistemas de control, es imprescindible el apoyo y compromiso de todos quienes conforman las organizaciones, por lo que su difusión y mejora continua deben conseguir la innovación de procesos y procedimientos. En un sentido más amplio la auditoría se debe aplicar como una "herramienta con unas potencialidades muy amplias, con gran utilidad para la dirección de la empresa como grupo de interés principal y directo, pero también con posibles aportaciones indirectas para otros actores inmersos en el día a día de la organización" (Calvo, Sigalat y Rodríguez, 2017, p. 232).

Dependiendo de quién realice las evaluaciones de control, el ámbito de aplicación varía, por lo que no debe entenderse bajo ningún precepto que hay 
Interrelación actual entre Gobierno Corporativo, Respondabilidad y Auditoría Integral: una visión reformulada

únicamente un enfoque financiero. Otro gran campo que debe ser evaluado permanentemente en las organizaciones son sus procesos productivos y administrativos, a fin de constatar su eficiencia y efectividad, comúnmente se le denomina auditoría operacional, administrativa o de desempeño, entendiendo que éstas a su vez deberían o podrían estar abarcadas por auditoría interna. Arens et al. (2007) determinan que la efectividad se refiere a la consecución de objetivos, en tanto que la eficiencia se refiere a los recursos utilizados para alcanzarlos. Un ejemplo de efectividad es la producción de partes sin defectos. La eficiencia tiene que ver con que esas partes se produzcan a un costo mínimo. Muñiz (2012), determina que un correcto sistema de control de gestión debe permitir conocer cómo, cuándo y dónde se han empleado los recursos de la organización (humanos, técnicos, productivos, comerciales, etc.) puestos a la disposición de los diferentes responsables (staff gerencial) para poder obtener resultados concretos en función de los objetivos planificados.

Obispo y Gonzales (2015), estipulan que los auditores internos contribuyen a asegurar la integridad de la estructura de controles internos de la administración y asisten a la gestión administrativa en el cumplimiento de sus responsabilidades. Los auditores financieros externos brindan a los usuarios de los estados financieros del ente auditado la seguridad independiente que necesitan: que se puede confiar en los datos en esos estados financieros. Los auditores del desempeño, tanto externos como internos, contribuyen a asegurar que los programas, actividades y funciones se manejen de manera eficiente y eficaz. La auditoría permite que las administraciones marquen la brecha o el contraste entre lo que es y lo que debería ser al hacerlo. Por lo que con una óptica más incluyente se puede aseverar que "la auditoría integral está basada en un enfoque interdisciplinario, que comprende aspectos legales, financieros, administrativos, operacionales, informáticos, entre otros, lo cual goza de aceptación en las organizaciones" (Sotomayor, 2008, p. 20).

Por otro lado, García-Marzá (2005), determina que la auditoría ética es un recurso básico para el diálogo entre todos los actores involucrados, no solo en la 
resolución de conflictos entre diferentes intereses, sino también para la discusión de estos conflictos y la búsqueda de acuerdos para evitar que surjan. En este sentido, se puede decir que la auditoría ética contribuye a la gestión del riesgo en la empresa. Se debe tomar en consideración que "la administración de riesgos corporativos se ocupa de los riesgos y oportunidades que afectan a la creación de valor o su preservación" (COSO, 2005, p. 4), ayuda a asegurar una información efectiva y el cumplimiento de leyes y regulaciones, además de ayudar a evitar daños a la reputación de la entidad y sus consecuencias derivadas. Más, se debe tomar en cuenta que la auditoría ética no se dirige tanto a los impactos como a la verificación y evaluación del funcionamiento del programa de ética y cumplimiento. El objetivo de la auditoría ética es recoger y evaluar los niveles de compromiso y aceptación de los grupos de interés que caracterizan a una gestión como ética (García-Marzá, 2017).

Se debe considerar que el proceso globalizador del entorno actual en donde se encuentran inmersas las empresas requiere que las organizaciones vayan sistematizando su operatividad, llegando a ocupar la tecnología un sitial que no puede ser desplazado, a la vez de constituirse en una enorme ventaja competitiva. Por lo que las operaciones de control también deben ser tecnificadas y adaptarse a los requerimientos institucionales. Dai y Vasarhelyi (2016), estipulan que las técnicas de análisis de auditoría se emplean para construir modelos sobre las bases de datos con el fin de monitorear la calidad del producto, identificar las fallas de las máquinas, optimizar costos y facilitar la toma de decisiones. El control en gran parte debería encontrarse automatizado. El proceso de auditoría depende en gran medida de una representación mundial simétrica de los procesos y de una fuerte interconexión analítica no solo de vínculos financieros, sino especialmente de vínculos no financieros con financieros.

\section{4.) Interrelación existente entre Auditoría Integral, Accountability y Gobierno Corporativo}


Interrelación actual entre Gobierno Corporativo, Respondabilidad y Auditoría Integral: una visión reformulada

El alcance de auditoría integral ya sea por la globalización, emisión de normativa internacional, avances tecnológicos o necesidad de ética global, ha variado y se ampliado drásticamente. "Los complejos desafíos mundiales en las organizaciones reclaman la participación de numerosos actores que aporten nuevas perspectivas y enfoques, donde se establezcan niveles para poder alcanzar el desarrollo humano sustentable" (Pertúz, 2018, 378). Se debe entender que el marco normativo contable como tal, consiste en una multiplicidad de elementos, incluyendo reglas multifacéticas y normas sobre autorizaciones presupuestarias, pagos de bonos, objetivos de desempeño y sistemas de rendición de cuentas (Englund, Gerdin y Abrahamsson, 2013). Por lo que el nivel de riesgo existente en el entorno actual, donde las organizaciones desarrollan sus actividades se debe contextualizar no solamente desde la óptica contable financiera, sino con una visión mucho más holística a fin de que dichos marcos normativos implementados constituyan un verdadero apoyo a las organizaciones que ayuden a fomentar la versatilidad de sus operaciones, con el valor agregado que se encuentren debidamente controladas y sistematizadas.

Por otro lado, una realidad existente es que la corrupción gira en torno a la actividad económica de la Administración; a la atribución de potestades de decisión, control y aplicación de fondos públicos a cargos políticos, funcionarios y personal contratado (Carbajo, 2012). La visión organizacional de la corrupción deja claro que esta es un fenómeno social de relaciones densas, donde lo considerado normal y anormal se negocian e intersecan en espacios donde sus intervinientes terminan interactuando en el día a día (Arellano, 2017). Justamente en esa interacción de funcionarios con procesos, actividades operativas, relaciones comerciales interinstitucionales, donde los protagonistas deben tener un marco claramente definido, se requiere un control permanente, a lo que debe incorporarse informes sobre los resultados obtenidos en base a las decisiones ejecutadas.

El enfoque de accountability es un elemento inclusivo de valor agregado en el ejercicio profesional del auditor, ya que puede ser considerado como una obligación 
ética y moral de rendir cuentas por el desempeño y resultado de la gestión de un funcionario (Rozas, 1998). A su vez, tiene como base la verificación integral de aspectos financieros, sin limitar su ámbito de aplicación a dicha evaluación. Se debe tomar en consideración que la administración de la compañía controla las principales fuentes de ingresos y ganancias volátiles de esta, a través de la administración de los riesgos subyacentes de negocios, operaciones, crédito y mercado. La administración también es responsable de las numerosas estimaciones y juicios subyacentes de los resultados informados (AICPA, 2016).

Por lo expuesto, se puede deducir claramente que no sea posible que exista un enfoque actual de auditoría integral sin que exista una adopción de un sistema de gobierno corporativo eficaz y rendición de cuentas permanente. El desafío de la auditoría integral radica en adoptar una perspectiva innovadora, basado en la autocrítica que permita ubicar a la profesión a la vanguardia de los hechos o simplemente ignorarlos, en cuyo caso se corre el riesgo de ser relegados por los stakeholders. Los reportes corporativos emitidos por auditoría deben ser mucho más eficientes, transparentes y confiables, para que permitan tomar decisiones de inversión mejor informadas (FEE, 2014).

\section{Consideraciones finales}

Las crisis están presentes en el transcurso de las actividades cotidianas en la sociedad contemporánea, de hecho, se pueden advertir los mismos problemas por el incremento generalizado de los niveles de corrupción tanto en el sector público, como en el sector privado, hasta de cierta manera de forma cíclica. La transparencia en la información emitida por las organizaciones empresariales beneficia a la sociedad, ya que permite la distribución más eficiente de los recursos. Por lo tanto, Auditoría Integral tiene por objetivo: mejorar tanto la calidad de la información corporativa como garantizar y hacer que los servicios de aseguramiento y consulta sean lo más relevantes posible. 
Interrelación actual entre Gobierno Corporativo, Respondabilidad y Auditoría Integral: una visión reformulada

La rendición de cuentas tiene una aplicación universal en el sentido que debería aplicarse en todas las esferas del conocimiento, tanto en el sector público, como en el sector privado. Acotando que al igual que gobierno corporativo, el resultado final de su adecuada implementación y adopción se traduce en términos de transparencia, no solamente a nivel empresarial, sino en términos mucho más amplios que abarcan el desarrollo de la sociedad.

En virtud de lo expuesto en el desarrollo de las descripciones realizadas, se puede determinar que Auditoría Integral tiene un ámbito de aplicación holístico y se plantean constantemente diferentes aspectos que deben ser considerados, con la finalidad de obtener una concepción integradora e innovadora que debería adoptar la alta gerencia, donde se fusione dichos elementos: gobierno corporativo y accountability, inmersos en un nuevo enfoque de auditoría integral, estableciéndose por tanto una relación inherente. En consecuencia, los sistemas bajo los cuales las corporaciones son administradas, el conjunto de políticas formales e informales que regulan las relaciones de los stakeholders con las mismas y la rendición de cuentas como medidas fehacientes de transparencia, conforman una amalgama que debe estar presente de manera sistemática y coordinada mediante una evaluación permanente que es la Auditoría Integral. El desafío se encuentra vigente y la lucha contra la corrupción es una tarea que nos compete a todos.

\section{Referencias Bibliográficas}

American Institute of Certified Public Accountants (AICPA) (2016). Management Override of Internal Control: The Achilles' Heel of Fraud Prevention. [Documento en Línea]. Extraído de http://www.aicpa.org/ForThePublic/AuditCommitteeEffectiveness/Download ableDocuments/achilles_heel.pdf consulta: 18/06/2018.

Arcot, Sridhar., Bruno, Valentina., Faure-Grimaud, Antoine (2010). Corporate governance in the UK: Is the comply or explain approach working? International Review of Law and Economics. Volumen 30, No. 2. Estados Unidos (Pp. 193-201). 
Arellano, David (2017). Corrupción como proceso organizacional: comprendiendo la lógica de la desnormalización de la corrupción. Contaduría y Administración. Volumen 62, No. 3. México (Pp. 810-826).

Arens, Alvin., Elder, Randal., Beasley, Mark (2007). Auditoría. Un enfoque integral. Pearson. México.

Bortolon, Patricia., Sarlo, Alfredo., Santos, Thaís (2013). Audit Costs and Corporate Governance. Revista Contabilidade \& Finanças. Volumen 24, No. 61. Brasil (Pp. 27-36).

Cadbury Committee (1992). Report of the Committee on the Financial Aspects of Corporate Governance. [Documento en Línea]. Extraído de http://www.ecgi.org/codes/documents/cadbury.pdf consulta: 18/06/2018.

Calvo, Ricard., Sigalat, Enric., Rodríguez, Juan (2017). La auditoría socio laboral y sus grupos de interés: ¿intereses comunes? ¿intereses compartidos? Una aproximación al caso valenciano. Lan Harremanak. Revista de Relaciones Laborales. No. 38. España (Pp. 229-258).

Caridad, Migdalia., Hernández, Pedro., De Pelekais, Cira (2014). Respondabilidad gerencial: elemento integrador de la sustentabilidad en la responsabilidad social empresarial. Revista Opción. Volumen 30, No. 75. Venezuela (Pp. 3554).

Carbajo, Fernando (2012). Corrupción en el sector privado (I): la corrupción privada y el derecho privado patrimonial. Revista Iustitia. No. 10. Colombia (Pp. 281342).

Claessens, Stijn., Yurtoglu, Burcin (2012). Corporate Governance and Development - An Unpdate. The International Finance Corporation. Estados Unidos.

Committee of Sponsoring Organization of the Treadway Commission (COSO) (2013). Control Interno - Marco integrado. Marco y apéndices. COSO. Estados Unidos.

Committee of Sponsoring Organization of the Treadway Commission (COSO) (2005). Administración de Riesgos Corporativos - Marco Integrado. COSO. Estados Unidos.

Dai, Jun., Vasarhelyi, Miklos (2016). Imagineering Audit 4.0. Journal of Emerging Technologies in Accounting. Volumen 13, No. 1. Estados Unidos (Pp. 1-15).

De La Torre, Mauricio (2017). Responsabilidad Social Corporativa y Auditoría Interna, una exigencia global imperativa. Revista Metanoia. Volumen 3, No. 3. Ecuador (Pp. 113-126).

Díaz, Nubia., Pineda, Eduardo., Reyes, José (2013). Respondabilidad en las actuaciones profesionales del contador público. Revista COEPTUM. Volumen 5, No. 2. Venezuela (Pp. 105-122).

Donleavy, Gabriel (2016). An Introduction to Accounting Theory. [Documento en Línea]. Extraído de https://bookboon.com/en/an-introduction-to-accountingtheory-ebook consulta 15/06/2018. 
Interrelación actual entre Gobierno Corporativo, Respondabilidad y Auditoría Integral: una visión reformulada

Englund, Hans., Gerdin, Jonas., Abrahamsson, Gun (2013). Accounting ambiguity and structural change. Accounting, Auditing \& Accountability Journal. Volumen 26, No. 3. United Kingdom (Pp. 423-448).

Federation of European Accountants (FEE) (2014). Opening a discussion: The Future of Audit and Assurance. Fédération des Experts-comptables Européens. Bélgica.

Fukuyama, Francis (2017). Corruption as a Political Phenomenon. En Kaushik Basu., Tito Cordella (Editores). Institutions, Governance and the Control of Corruption. Palgrave Macmillan. Estados Unidos.

García, Carlos., Socorro, Cecilia., Fernández, Johender (2014). Respondabilidad como herramienta de control de la información financiera. Revista TELOS. Volumen 16, No. 3. Venezuela (Pp. 447-462).

García-Marzá, Domingo (2017). De los códigos a las auditorías éticas: una infraestructura ética para la comunicación de la responsabilidad social. El profesional de la información. Volumen 26, No. 2. España (Pp. 268-276).

García-Marzá, Domingo (2005). Trust and Dialogue: Theoretical Approaches to Ethics Auditing. Journal of Business Ethics. Volumen 57, No. 3. Países Bajos (Pp. 209-219).

Gunz, Sally (2012). Accounting and Business Ethics. En Ruth Chadwick (Editor). Encyclopedia of Applied Ethics (Second Edition). Academic Press. Estados Unidos.

Kliksberg, Bernardo (2012). Un examen de las relaciones entre ética y economía. Algunas anotaciones para la acción. En BBVA (Editores). Valores y ética para el siglo XXI. Editorial BBVA. España.

Kolb, Robert (2008). Encyclopedia of Business Ethics and Society (volumen cinco). Editorial Sage. Estados Unidos.

Maldonado, Milton (2016). Revolución Contable: para la Justicia Social y la Protección Ambiental. [Documento en Línea]. Extraído de http://liderazgoyriesgos.com/Revolucion.html consulta: 10/06/2018.

Mostepaniuk, Alla (2017). Corporate Governance. En Lawrence Emeagwali (Editor). Corporate Governance and Strategic Decision Making. InTechOpen. United Kingdom.

Muñiz, Luis (2012). Cómo implantar y evaluar un sistema de Control de Gestión. Editorial Profit. España.

Obispo, Daniel., Gonzales, Yuri (2015). Caracterización del control interno en la gestión de las empresas comerciales del Perú 2013. In Crescendo. Volumen 6, No. 1. Perú (Pp. 64-73).

Organización para la Cooperación y el Desarrollo Económicos (OCDE) (2016). Principios de Gobierno Corporativo de la OCDE y del G20. [Documento en Línea]. Extraído de http://dx.doi.org/10.1787/9789264259171-es consulta: 15/06/2018. 
Pertúz, Fredy (2018). Liderazgo Transformacional en Empresas Sociales Desde la perspectiva Ética de la Responsabilidad Social Empresarial. Revista TELOS. Volumen 20, No. 2. Venezuela (Pp. 377-400).

Rozas, Alan (1998). Accountability ("Respondabilidad"). Revista Yupaq. Volumen 2, No. 5. Perú (Pp. 21-22).

Shleifer, Andrei., Vishny, Robert (1997). A Survey of Corporate Governance. The Journal of Finance. Volumen 52, No. 2. Estados Unidos (Pp. 737-783).

Søreide, Tina (2006). Corruption in international business transactions: the perspective of Norwegian firms. En Susan Rose-Ackerman (Editor). International Handbook on the Economics of Corruption. Edward Elgar. Estados Unidos. Sotomayor, Alfonso (2008). Auditoría Administrativa. Proceso y aplicación. McGrawHill. México.

Torres, Adriana., Negrón, Alexander (2016). La respondabilidad gerencial como herramienta de competitividad empresarial. Revista Electrónica Arbitrada del Centro de Investigaciones de Ciencias Administrativas y Gerenciales. Volumen 13, No. 1. Venezuela (Pp. 130-147).

Yoris, Onás (2017). Gerencia del No Equilibrio: Tendencia Disruptiva en las Organizaciones del Siglo XXI. Revista TELOS. Volumen 19, No. 2. Venezuela (Pp. 331-342). 